\title{
М.К. Хасанов
}

\section{МАТЕМАТИЧЕСКАЯ МОДЕЛЬ ИНЖЕКЦИИ СЕРОВОДОРОДА В ПРИРОДНЫЙ ПЛАСТ, СОПРОВОЖДАЮЩЕЙСЯ ОБРАЗОВАНИЕМ ЕГО ГАЗОГИДРАТА ${ }^{1}$}

\begin{abstract}
На основе уравнений механики многофазных сред построена математическая модель инжекции жидкого сероводорода в пористый пласт, насыщенный нефтью и водой. Построены автомодельные решения задачи для случая, когда в пласте возникают две подвижные межфазные поверхности, на которых происходят образование газогидрата сероводорода и вытеснения нефти сероводородом. Исследована зависимость автомодельных координат межфазных границ от проницаемости пласта и его начального давления. Установлено, что при низких значениях проницаемости и высоких значениях начального давления пласта может происходить слияние межфазных поверхностей. Найдены зависимости критических значений давления инжекции, соответствующих слиянию межфазных поверхностей, от проницаемости пласта и его исходного давления.
\end{abstract}

Ключевые слова: математическая модель, автомодельное решение, пористая среда, фильтрачия, газогидраты, сероводород.

Одним из методов снижения эмиссии сероводорода, вырабатываемого промышленными предприятиями, в атмосферу является его подземное захоронение в истощенных нефтегазовых коллекторах $[1,2]$. Поскольку при долгосрочном подземном хранении утилизируемых газов в виде флюида существует риск их утечки на поверхность, то рассматривается возможность их перевода в газогидратное состояние, позволяющее по сравнению со свободным состоянием хранить одинаковые объемы газа при меньших давлениях [3-5].

Поскольку любые технологические идеи должны быть подкреплены соответствующими расчетами, основанными на теоретических моделях, то актуальной задачей является построение адекватных математических моделей гидратообразования в природных пластах. Математические модели образования газогидратов в пористых средах, насыщенных метаном и водой, сформулированы, в частности, в работах $[6,7]$. Математические модели образования газогидрата $\mathrm{H}_{2} \mathrm{~S}$ в пластах, насыщенных нефтью и водой, при инжекции жидкого сероводорода представлены в работах $[8,9]$. Однако в данных работах задача решена в наиболее простой постановке, когда образование газогидрата происходит на границе, совпадающей с границей вытеснения нефти сероводородом. В настоящей работе построена математическая модель образования газогидрата $\mathrm{H}_{2} \mathrm{~S}$ для случая, когда гидратообразование происходит на фронтальной границе, которая не совпадает с границей вытеснения нефти жидким сероводородом.

\footnotetext{
${ }^{1}$ Работа выполнена при финансовой поддержке РФФИ и Республики Башкортостан (проект 17-48020123 p_a).
} 


\section{Постановка задачи}

Термобарические условия существования газогидрата $\mathrm{H}_{2} \mathrm{~S}$ показаны на фазовой диаграмме (рис. 1) [5]. На данной диаграмме кривая 1 определяет трехфазное равновесие между газообразным сероводородом, водой и газогидратом $\mathrm{H}_{2} \mathrm{~S}$, кривая 2 - равновесие между жидким сероводородом, водой и газогидратом $\mathrm{H}_{2} \mathrm{~S}$, a кривая 3 - двухфазное равновесие между газообразным и жидким сероводородом. Соответственно газогидрат $\mathrm{H}_{2} \mathrm{~S}$ существует левее кривых 1 и 3 . В квадрупольной точке $Q\left(T_{Q}=302.6 \mathrm{~K}\right.$ и $\left.p_{Q}=2.24 \mathrm{MПа}\right)$ все четыре указанные фазы находятся в равновесии.

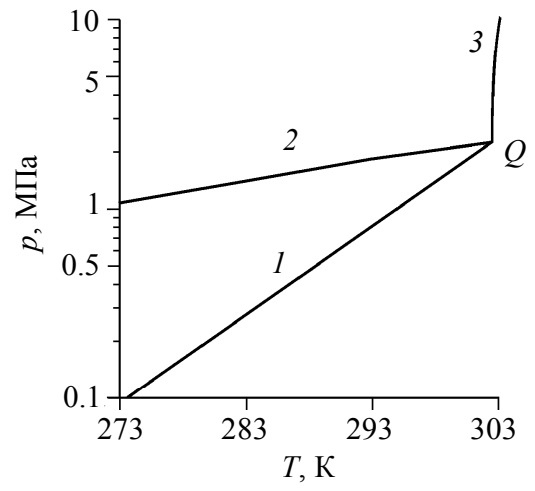

Рис. 1. Фазовая диаграмма системы «сероводород - вода»

Fig. 1. Phase diagram of the system "hydrogen sulfide - water"

Пусть полубесконечный горизонтальный пористый пласт (занимающий полупространство $x>0$ ) в начальный момент времени насыщен водой и нефтью. Будем полагать, что начальная температура пласта $T_{0}$ выше температуры $T_{Q}$, соответствующей квадрупольной точке. Следовательно, в рассматриваемой задаче начальное состояние пласта не соответствует условиям образования газогидрата сероводорода. Положим, что через границу $(x=0)$ закачивается жидкий сероводород, давление $p_{e}$ и температура $T_{e}$ которого соответствуют условиям существования газогидрата сероводорода.

В данной работе будем рассматривать модель с поршневым вытеснением нефти жидким сероводородом, а также случай, когда значение исходной водонасыщенности пласта не превышает 0.2 , тогда воду можно считать неподвижной. Таким образом, в данной модели только две фазы являются подвижными - нефть и жидкий сероводород. Рассматривая масштабы времени, значительно превышающие характерное время кинетики процесса гидратообразования, и учитывая, что в рассматриваемой задаче начальное состояние пласта не соответствует условиям образования газогидрата сероводорода можно полагать, что гидратообразование происходит на фронтальной границе, не совпадающей с границей вытеснения нефти сероводородом. Следовательно, в рассматриваемом случае в пласте образуются три характерные области (рис. 2). В первой (ближней) области поры насыщены сероводородом и газогидратом $\mathrm{H}_{2} \mathrm{~S}$, во второй (промежуточной) области присутствуют вода и сероводород, а в третьей (дальней) зоне поры насыщены водой и нефтью. Соответственно существуют две подвижные межфазные поверхно- 
сти: между первой и второй областями, где вода полностью переходит в газогидратное состояние (фронт гидратообразования), и между второй и третьей областями, где происходит вытеснение нефти сероводородом (фронт вытеснения).

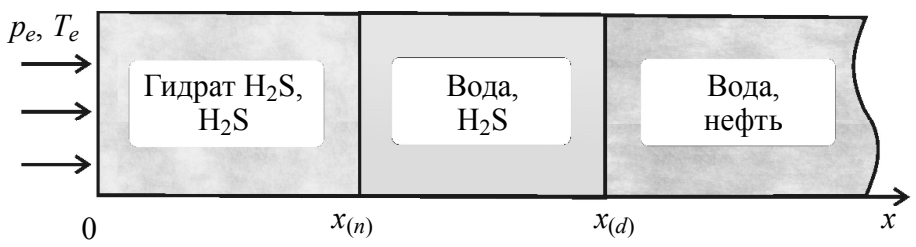

Рис. 2. Схема задачи

Fig. 2. Schematic diagram of the problem

\section{Основные уравнения}

Примем следующие упрощающие предположения: пористость пласта $m$ постоянна, скелет пористой среды, газогидрат и вода несжимаемы и неподвижны. Газогидрат $\mathrm{H}_{2} \mathrm{~S}$ является двухкомпонентной системой с массовой концентрацией сероводорода $G$. Жидкий сероводород и нефть будем считать слабосжимаемыми жидкостями.

Система основных уравнений, описывающая в одномерном случае процессы фильтрации и теплопереноса в пористом пласте представляет собой законы сохранения масс и энергии, закон Дарси и уравнения состояния нефти и воды [10]:

$$
\begin{gathered}
\frac{\partial}{\partial t}\left(\rho_{i} m S_{i}\right)+\frac{\partial}{\partial x}\left(\rho_{i} m S_{i} \mathrm{v}_{i}\right)=0, \\
\rho c \frac{\partial T}{\partial t}+\rho_{i} c_{i} m S_{i} \mathrm{v}_{i} \frac{\partial T}{\partial x}=\frac{\partial}{\partial x}\left(\lambda \frac{\partial T}{\partial x}\right), \\
m S_{i} \mathrm{v}_{i}=-\frac{k_{i}}{\mu_{i}} \frac{\partial p}{\partial x}, \\
\rho_{i}=\rho_{0 i}\left(1+\beta_{i}\left(p-p_{0}\right)\right) .
\end{gathered}
$$

Здесь $t$ - время; $x$-координата; $m$ - пористость; $T$ - температура; $p$ - давление; нижние индексы $i=l, s$ относятся соответственно к параметрам нефти и сероводорода; $\rho_{i}-$ плотность; $v_{i}$ - действительная средняя скорость; $k_{i}-$ фазовая проницаемость; $\mu_{i}$ - динамическая вязкость; $c_{i}$ - удельная теплоемкость; $S_{i}$ - насыщенность; $\beta_{i}-$ коэффициент сжимаемости; $\rho c$ и $\lambda$ - эффективные значения объемной теплоемкости и коэффициента теплопроводности насыщенного пласта, которые считаем постоянными величинами (так как основной вклад в их значения вносят параметры горной породы).

Зависимость коэффициента фазовой проницаемости $k_{i}$ от насыщенности $S_{i}$ и абсолютной проницаемости пласта $k_{0}$ зададим следующим образом:

$$
k_{i}=k_{0} S_{i} \quad(i=s, l) .
$$

На поверхности $x=x_{(n)}$, разделяющей первую и вторую области, происходит полный переход воды в гидратное состояние. Поэтому из условий баланса массы 
и тепла на этой границе следует

$$
\begin{gathered}
-\frac{k_{s(1)}}{\mu_{s}} \frac{\partial p_{(1)}}{\partial x}+\frac{k_{s(2)}}{\mu_{s}} \frac{\partial p_{(2)}}{\partial x}=m S_{h}\left(\frac{\rho_{h} G}{\rho_{0 s}}+\frac{\rho_{h}(1-G)}{\rho_{w}}-1\right) V_{(n)}, \\
m S_{h} \rho_{h}(1-G) V_{(n)}=m S_{w 0} \rho_{w} V_{(n)}, \\
\lambda \frac{\partial T_{(1)}}{\partial x}-\lambda \frac{\partial T_{(2)}}{\partial x}=m S_{h} \rho_{h} L_{h} V_{(n)} .
\end{gathered}
$$

Здесь $V_{(n)}$ - скорость движения фронта образования газогидрата $\mathrm{H}_{2} \mathrm{~S}, G$ - массовая концентрация сероводорода в гидрате, $L_{h}$ - удельная теплота образования газогидрата $\mathrm{H}_{2} \mathrm{~S}, S_{w 0}$ - начальная водонасыщенность пористой среды, $\rho_{w}-$ плотность воды, $k_{s(1)}$ - фазовая проницаемость для сероводорода в первой области, $k_{s(2)}-\phi а-$ зовая проницаемость для сероводорода во второй области. Нижний индекс $n$ относится к параметрам на границе, разделяющей первую и вторую области, а нижние индексы 1 и 2 - к параметрам первой и второй областей соответственно. Температуру на этой границе будем считать непрерывной и равной температуре квадрупольной точки $T_{\mathrm{Q}}$.

На поверхности $x=x_{(d)}$, разделяющей вторую и третью области, происходит вытеснение нефти сероводородом. Поэтому с учетом условий баланса массы нефти и сероводорода, а также баланса тепла на этой границе имеем

$$
\begin{gathered}
-\frac{k_{s(2)}}{\mu_{s}} \frac{\partial p_{(2)}}{\partial x}=m\left(1-S_{w 0}\right) V_{(d)} \\
-\frac{k_{l}}{\mu_{l}} \frac{\partial p_{(3)}}{\partial x}=m\left(1-S_{w 0}\right) V_{(d)} \\
\lambda \frac{\partial T_{(2)}}{\partial x}-\lambda \frac{\partial T_{(3)}}{\partial x}=0
\end{gathered}
$$

где $V_{(d)}$ - скорость движения фронта вытеснения нефти сероводородом, $k_{l}$ - фазовая проницаемость для нефти в третьей области. Нижний индекс $d$ относится к параметрам на границе, разделяющей вторую и третью области, а нижний индекс 3 - к параметрам третьей области.

На обеих границах давление и температуру будем считать непрерывными величинами.

Из уравнения (3) можно найти величину гидратонасыщенности в первой области:

$$
S_{h}=\frac{\rho_{w} S_{w 0}}{\rho_{h}(1-G)} .
$$

На основе уравнений системы (1), уравнения пьезопроводности и температуропроводности запишутся в виде

$$
\frac{\partial p_{(j)}}{\partial t}=\chi_{(j)}^{(p)} \frac{\partial}{\partial x}\left(\frac{\partial p_{(j)}}{\partial x}\right), j=1,2,3 ;
$$




$$
\frac{\partial T_{(j)}}{\partial t}=\chi^{(T)} \frac{\partial}{\partial x}\left(\frac{\partial T_{(j)}}{\partial x}\right)+\frac{1}{2} \chi^{(T)} \alpha_{(j)} \frac{\partial p_{(j)}}{\partial x} \frac{\partial T}{\partial x}, j=1,2,3,
$$

где $\quad \chi_{(1)}^{(p)}=\frac{k_{s(1)}}{\mu_{s} m\left(1-S_{h}\right) \beta_{s}}, \chi_{(2)}^{(p)}=\frac{k_{s(2)}}{\mu_{s} m\left(1-S_{w 0}\right) \beta_{s}}, \chi_{(3)}^{(p)}=\frac{k_{l}}{\mu_{l} m\left(1-S_{w 0}\right) \beta_{l}}$,

$$
\chi^{(T)}=\frac{\lambda}{\rho c}, \alpha_{(1)}=\frac{2 \rho_{0 s} c_{s} k_{s(1)}}{\lambda \mu_{s} \beta_{s}}, \alpha_{(2)}=\frac{2 \rho_{0 s} c_{s} k_{s(2)}}{\lambda \mu_{s} \beta_{s}}, \alpha_{(3)}=\frac{2 \rho_{0 l} c_{l} k_{l}}{\lambda \mu_{l} \beta_{l}},
$$

нижние индексы $j=1,2,3$ относятся к параметрам соответственно первой, второй и третьей областей.

Поскольку в рассматриваемой задаче перепады температур в пласте незначительны $\left(\Delta T<<T_{0}\right)$, то в уравнении пьезопроводности отброшено слагаемое, отвечающее за переменность температуры.

Начальные и граничные условия имеют вид

$$
\begin{gathered}
t=0: \quad S_{w}=S_{w 0}, T=T_{0}, \quad p=p_{0} \quad(x \geq 0) . \\
x=0: T=T_{e}, \quad p=p_{e} \quad(t>0) .
\end{gathered}
$$

\section{Автомодельное решение}

Введем автомодельную переменную: $\xi=x / \sqrt{\chi^{(T)} t}$. Для этой переменной уравнения пьезопроводности и температуропроводности $(8),(9)$ в каждой области примут вид

$$
\begin{gathered}
-\xi \frac{d p_{(j)}}{d \xi}=2 \eta_{(j)} \frac{d}{d \xi}\left(\frac{d p_{(j)}}{d \xi}\right) ; \\
-\xi \frac{d T_{(j)}}{d \xi}=\alpha_{(j)} \frac{d p_{(j)}}{d \xi} \frac{d T_{(j)}}{d \xi}+2 \frac{d}{d \xi}\left(\frac{d T_{(j)}}{d \xi}\right),
\end{gathered}
$$

где $\eta_{(j)}=\chi_{(j)}^{(p)} / \chi^{(T)}$.

После интегрирования (11), (12) с учетом начальных и граничных условий (10) решения для распределения давления и температуры в каждой из областей могут быть записаны в виде

$$
\begin{gathered}
p_{(1)}=p_{(n)}+\frac{\left(p_{\mathrm{e}}-p_{(n)}\right) \mathrm{I}\left(\xi, \xi_{(n)} ; \eta_{(1)}\right)}{\mathrm{I}\left(0, \xi_{(n)} ; \eta_{(1)}\right)}, T_{(1)}=T_{(n)}+\frac{\left(T_{e}-T_{(n)}\right) \mathrm{J}_{(1)}\left(\xi, \xi_{(n)}\right)}{\mathrm{J}_{(1)}\left(0, \xi_{(n)}\right)} \\
p_{(2)}=p_{(d)}+\frac{\left(p_{(n)}-p_{(d)}\right) \mathrm{I}\left(\xi, \xi_{(d)} ; \eta_{(2)}\right)}{\mathrm{I}\left(\xi_{(n)}, \xi_{(d)} ; \eta_{(2)}\right)}, T_{(2)}=T_{(d)}+\frac{\left(T_{(n)}-T_{(d)}\right) \mathrm{J}_{(2)}\left(\xi_{,}, \xi_{(d)}\right)}{\mathrm{J}_{(2)}\left(\xi_{(n)}, \xi_{(d)}\right)} \\
p_{(3)}=p_{0}+\frac{\left(p_{(d)}-p_{0}\right) \mathrm{I}\left(\xi, \infty ; \eta_{(3)}\right)}{\mathrm{I}\left(\xi_{(d)}, \infty ; \eta_{(3)}\right)}, T_{(3)}=T_{0}+\frac{\left(T_{(d)}-T_{0}\right) \mathrm{J}_{(3)}(\xi, \infty)}{\mathrm{J}_{(3)}\left(\xi_{(d)}, \infty\right)}
\end{gathered}
$$


где введены обозначения интегралов:

$$
\mathrm{I}(a, b ; c)=\int_{a}^{b} \exp \left(-\frac{\xi^{2}}{4 c}\right) d \xi ; \quad \mathrm{J}_{(j)}(a, b)=\int_{a}^{b} \exp \left(-\frac{\xi^{2}}{4}-\alpha_{(j)} p_{(j)}\right) d \xi ;
$$

$p_{(n)}$ и $T_{(n)}$ - давление и температура на границе между первой и второй областями; $p_{(d)}$ и $T_{(d)}$ - давление и температура на границе между второй и третьей областями.

На основе соотношений (2), (4) с учетом решений (13), (14) получим уравнения для определения координаты фронта гидратообразования $\xi_{(n)}$ и значений параметров на нем:

$$
\begin{gathered}
\left(p_{(d)}-p_{(n)}\right) \exp \left(-\frac{\xi_{(n)}^{2}}{4 \eta_{(2)}}\right) \\
k_{s(2)} \frac{\left(p_{(n)}-p_{e}\right) \exp \left(-\frac{\xi_{(n)}^{2}}{4 \eta_{(1)}}\right)}{\mathrm{I}\left(\xi_{(n)}, \xi_{(d)} ; \eta_{(2)}\right)}-k_{s(1)} \frac{\mathrm{I}\left(0, \xi_{(n)} ; \eta_{(1)}\right)}{\left.T_{(n)}-T_{e}\right) \exp \left(-\frac{\xi_{(n)}^{2}}{4}-\alpha_{(1)} p_{(1)}\right)}\left(T_{(d)}-T_{(n)}\right) \exp \left(-\frac{\xi_{(n)}^{2}}{4}-\alpha_{(2)} p_{(2)}\right) \\
\frac{\mathrm{J}_{(1)}\left(0, \xi_{(n)}\right) \xi}{\mathrm{J}_{(2)}\left(\xi_{(n)}, \xi_{(d)}\right)}=\frac{m \rho_{h} L_{h}}{2 \rho c} S_{h} \xi_{(n)} ; \\
T_{(n)}=T_{Q},
\end{gathered}
$$

где $K=m \mu_{s} \chi^{(T)}\left(\frac{\rho_{h} G}{\rho_{s 0}}+\frac{\rho_{h}(1-G)}{\rho_{w}}-1\right)$.

Аналогично на основе соотношений (5) - (7) с учетом решений (14) и (15) получим систему уравнений для определения координаты фронта вытеснения нефти сероводородом $\xi_{(\mathrm{d})}$ и значений параметров на нем:

$$
\begin{gathered}
k_{s(2)} \frac{\left(p_{(n)}-p_{(d)}\right) \exp \left(-\frac{\xi_{(d)}^{2}}{4 \eta_{(2)}}\right)}{\mathrm{I}\left(\xi_{(n)}, \xi_{(d)} ; \eta_{(2)}\right)}=m \mu_{s} \chi^{(T)}\left(1-S_{w 0}\right) \xi_{(d)} ; \\
k_{l} \frac{\left(p_{(d)}-p_{0}\right) \exp \left(-\frac{\xi_{(d)}^{2}}{4 \eta_{(3)}}\right)}{\left(T_{(d)}-T_{(n)}\right) \exp \left(-\frac{\xi_{(d)}^{2}}{4}-\alpha_{(2)} p_{(d)}\right)}=m \mu_{l} \chi^{(T)}\left(1-{S_{w 0}}_{(2)}\right) \xi_{(d)} ; \\
\frac{\mathrm{J}_{(2)}\left(\xi_{(n)}, \xi_{(d)}\right)}{T_{0}}-\frac{\left.T_{(d)}\right) \exp \left(-\frac{\xi_{(d)}^{2}}{4}-\alpha_{(3)} p_{(d)}\right)}{\mathrm{J}_{(3)}\left(\xi_{(d)}, \infty\right)}=0 .
\end{gathered}
$$

Система граничных уравнений (16) - (21) в работе решена следующим образом. Вначале задается нулевое приближение искомых величин на фронте гидратообразования. Далее, решая уравнение (19), находим величину $p_{(d)}$ (как функцию $\left.\xi_{(d)}\right)$, подставляя которую в уравнение (20) получим трансцендентное уравнение для нахождения $\xi_{(d)}$. Решив данное уравнение (методом половинного деления), 
определим величину $\xi_{(d)}$ (и соответственно $p_{(d)}$ ), а затем из $(21)$ определяем $T_{(d)}$. Далее, подставляя (18) в уравнение (17), получим трансцендентное уравнение для нахождения $\xi_{(n)}$. Решая данное уравнение (методом половинного деления), определяем новое приближение величины $\xi_{(n)}$. Затем, решая уравнение (16), находим новое приближение величины $p_{(n)}$. В результате циклического повторения описанной итерационной процедуры получаем последовательность приближенных значений, которая сходится к искомым значениям граничных параметров.

\section{Результаты расчетов}

На рис. 3 приведены распределения температуры и давления в зависимости от автомодельных переменных. Здесь и далее, если не оговорено иное, для параметров, характеризующих систему, приняты следующие значения: $m=0,1$, $S_{w 0}=0.2, \quad p_{e}=8.6 \mathrm{M \Pi a}, \quad T_{e}=290 \mathrm{~K}, \quad p_{0}=8 \mathrm{M \Pi a}, T_{0}=305 \mathrm{~K}, \quad k_{0}=10^{-11} \mathrm{M}^{2}$, $G=0.24, \quad \beta_{l}=1 \cdot 10^{-9} \Pi^{-1}, \quad \beta_{s}=3 \cdot 10^{-9} \Pi^{-1}, \quad \lambda=2 \mathrm{BT} /(\mathrm{M} \cdot \mathrm{K}), \quad \rho c=2 \cdot 10^{6}$ Дж/(К·Кг), $\mu_{l}=2 \cdot 10^{-3}$ Па.c, $\quad \mu_{s}=2 \cdot 10^{-4}$ Па $\cdot c, \quad \rho_{h}=1003 \kappa г / \mathrm{M}^{3}, \quad \rho_{w}=1000 \kappa \Gamma / \mathrm{M}^{3}, \quad \rho_{0 l}=900 \kappa \Gamma / \mathrm{M}^{3}$, $\rho_{0 s}=890$ кг $/ \mathrm{M}^{3}, c_{l}=1900$ Дж/(К·кг), $c_{\mathrm{s}}=1800$ Дж/(К·кг $), L_{h}=4.1 \cdot 10^{5}$ Дж/кг.

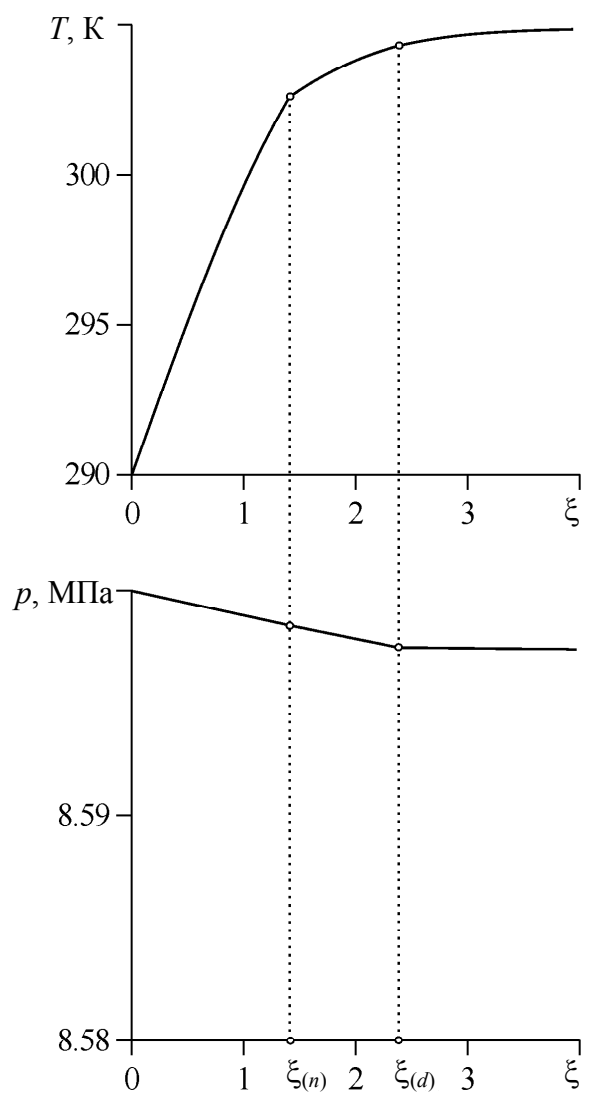

Рис. 3. Распределения температуры и давления

Fig. 3. Temperature and pressure distributions 
Рис. 3 показывает, что перераспределение давления в пласте происходит значительно быстрее изменения температуры. Это обусловлено тем, что величина коэффициента пьезопроводности $\chi^{(p)}=\frac{k}{\mu m \beta}$ значительно превышает величину коэффициента температуропроводности $\chi^{(T)}=\frac{\lambda}{\rho c}$. Кроме того, согласно рис. 3 , граница образования газогидрата движется очень медленно. Это обусловлено тем, что процесс образования газогидрата сопровождается выделением тепла, а температура на фронте гидратообразования не должна превышать температуру квадрупольной точки $T_{\mathrm{Q}}$. Поэтому движение границы образования газогидрата лимитируется скоростью отвода выделяющейся энергии, которая оттекает от фронта фазовых переводов в основном за счет теплопроводности в пласте. Вследствие того, что процесс образования газогидрата сопровождается выделением тепла, градиент температуры на ближней границе фазовых переходов согласно рис. 3 терпит разрыв.

На рис. 4 приведены зависимости координат фронтов образования газогидрата $\mathrm{H}_{2} \mathrm{~S}$ (кривая 1) и вытеснения нефти жидким сероводородом (кривая 2) от проницаемости пласта $(a)$ и его начального давления $(b)$. Из рис. 4 видно, что скорость фронта образования газогидрата $\mathrm{H}_{2} \mathrm{~S}$ практически не зависит от начального давления пласта и его проницаемости. Это обусловлено тем, что скорость данного фронта лимитируется, прежде всего, отводом тепла, выделяющегося при образовании газогидрата $\mathrm{H}_{2} \mathrm{~S}$. Также, согласно рис. 4 , скорость фронта вытеснения нефти сероводородом снижается с увеличением начального давления пласта и уменьшением его проницаемости. Это обусловлено тем, что скорость фронта вытеснения нефти сероводородом лимитируется скоростью течения в пласте, которая согласно закону Дарси пропорциональна перепаду давления в пласте и его проницаемости.
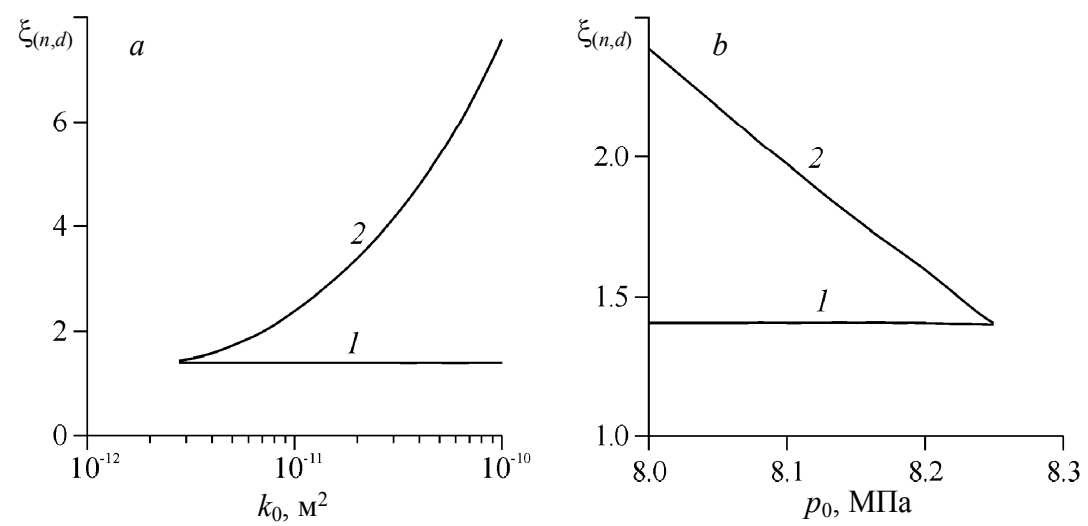

Рис. 4. Зависимость координат границ образования газогидрата $\mathrm{H}_{2} \mathrm{~S}$ (кр. 1) и вытеснения нефти сероводородом (кр. 2) от проницаемости пласта $(a)$ и начального давления $(b)$

Fig. 4. Coordinates of the boundaries of $\mathrm{H}_{2} \mathrm{~S}$ gas hydrate formation (curve 1) and displacement of oil by hydrogen sulfide (curve 2 ) as functions of $(a)$ permeability of the reservoir and $(b)$ initial pressure 
Также видно, что при достаточно больших значениях исходного давления пласта и его проницаемости происходит слияние фронтов. В этом случае режим, при котором гидратообразование происходит на фронтальной границе, а фронт вытеснения нефти сероводородом опережает данную границу, переходит в режим, при котором образование газогидрата сероводорода происходит на фронтальной границе, совпадающей с границей вытеснения нефти жидким сероводородом.

Рассматриваемый в данной работе режим реализуется в том случае, когда скорость фронта вытеснения нефти сероводородом (лимитируемая скоростью течения в пласте) превышает скорость фронта гидратообразования. Поэтому существует некоторое критическое значение давления инжекции сероводорода, ниже которого происходит слияние границ. На рис. 5 приведены зависимости величины данного давления от проницаемости при разных значениях исходного давления пласта $\left.p_{0}=7.9 \mathrm{MПа} \mathrm{(кривая} 1\right)$ и $p_{0}=8 \mathrm{MПа} \mathrm{(кривая} \mathrm{2).}$

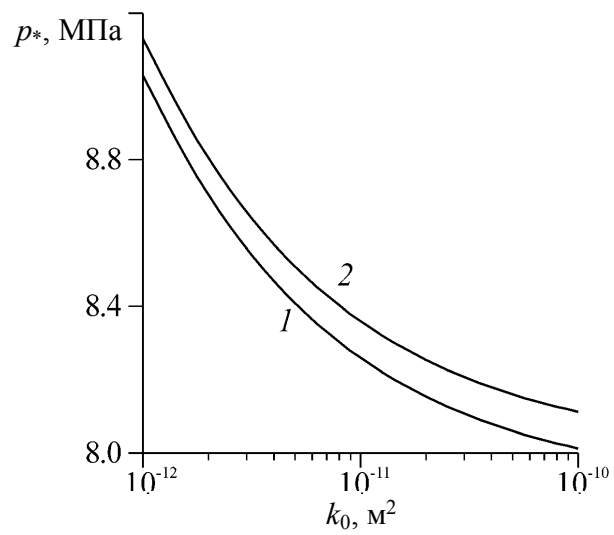

Рис. 5. Зависимость предельного давления от проницаемости пласта при $p_{0}=7.9 \mathrm{MПа} \mathrm{(кривая} \mathrm{1)} \mathrm{и} p_{0}=8 \mathrm{MПа} \mathrm{(кривая} \mathrm{2)}$

Fig. 5. Limiting pressure as a function of permeability of the reservoir at $p_{0}=(1) 7.9$ and (2) $8 \mathrm{MPa}$

Согласно рис. 5, с увеличением проницаемости пласта и уменьшением его начального давления критическое значение давления снижается. Это обусловлено тем, что с увеличением проницаемости и уменьшением начального давления пласта увеличивается интенсивность массопереноса в пласте и соответственно возрастает скорость фронта вытеснения нефти сероводородом. Поэтому в этом случаe данный фронт опережает границу образования газогидрата $\mathrm{H}_{2} \mathrm{~S}$ даже при малых значениях давления инжекции сероводорода.

\section{Заключение}

Предложена математическая модель закачки жидкого сероводорода в пласт, насыщенный нефтью и водой, сопровождающейся образованием газогидрата сероводорода. Рассмотрен случай, когда фронт вытеснения нефти сероводородом опережает фронт образования газогидрата $\mathrm{H}_{2} \mathrm{~S}$. Результаты расчетов показали, что скорость фронта вытеснения нефти сероводородом снижается с увеличением начального давления пласта и уменьшением его проницаемости. Поэтому при достаточно больших значениях исходного давления пласта и его проницаемости 
может происходить слияние фронтов образования газогидрата $\mathrm{H}_{2} \mathrm{~S}$ и вытеснения нефти сероводородом. Получена зависимость критического значения давления инжекции сероводорода, ниже которого происходит слияние фронтов, от проницаемости пласта. Установлено, что с увеличением проницаемости пласта и уменьшением его начального давления критическое значение давления снижается.

\section{ЛИТЕРАТУРА}

1. Machel H.G. Geological and hydrogeological evaluation of the Nisku Q-Pool in Alberta, Canada, for $\mathrm{H}_{2} \mathrm{~S}$ and/or $\mathrm{CO}_{2}$ storage // Oil Gas Sci. Technol. 2005. V. 60. P. 51-65. DOI: 10.2516/ogst:2005005.

2. Xu T., Apps J. A., Pruess K. and Yamamoto H. Numerical modeling of injection and mineral trapping of $\mathrm{CO}_{2}$ with $\mathrm{H}_{2} \mathrm{~S}$ and $\mathrm{SO}_{2}$ in a sandstone formation // Chem. Geology. 2007. V. 24. No 3-4. P. 319-346. DOI: 10.1016/j.chemgeo.2007.03.022.

3. Дучков А.Д., Соколова Л.С., Аюнов Д.Е., Пермяков М.Е. Оценка возможности захоронения углекислого газа в криолитозоне Западной Сибири // Криосфера Земли. 2009. Т. 13. № 4. С. 62-68.

4. Чувилин E.M., Гурьева О.М. Экспериментальное изучение образования гидратов $\mathrm{CO}_{2}$ в поровом пространстве промерзающих и мерзлых пород // Криосфера Земли. 2009. Т. 13. № 3. C. 70-79.

5. Бык С.Ш., Макогон Ю.Ф., Фомина В.И. Газовые гидраты. М.: Химия, 1980.

6. Шагапов В.Ш., Рафикова Г.Р., Хасанов М.К. К теории образования газогидрата в частично водонасыщенной пористой среде при нагнетании метана // Теплофизика высоких температур. 2016. Т. 54. № 6. C. 911-920. DOI: 10.1134/S0018151X16060171.

7. Гималтдинов И.К., Хасанов М.К. Математическая модель образования газогидрата при инжекции газа в пласт, частично насыщенный льдом // Прикладная математика и механика. 2016. Т. 80. № 1. С. 80-90. DOI: 10.1016/j.jappmathmech.2016.05.009.

8. Хасанов М.К. Инжекция жидкого сероводорода в пласт, насыщенный нефтью и водой // Вестник Тюменского государственного университета. Физико-математическое моделирование. Нефть, газ, энергетика. 2017. Т. 3. № 2. С. 72-84. DOI: 10.21684/2411-79782017-3-2-72-84.

9. Хасанов М.К. Математическая модель инжекции сероводорода в пористую среду, насыщенную нефтью и водой // Изв. ТулГУ. Технические науки. 2017. Вып. 9. Ч. 1. C. 337-345.

10. Shagapov V.Sh., Khasanov M.K., Musakaev N.G., Ngoc Hai Duong Theoretical research of the gas hydrate deposits development using the injection of carbon dioxide // International J. Heat and Mass Transfer. 2017. V. 107. P. 347-357. DOI: 10.1016/j.ijheatmasstransfer. 2016.11.034.

Статья поступила 06.02.2018 г.

Khasanov M.K. (2018) MATHEMATICAL MODEL OF HYDROGEN SULFIDE INJECTION INTO A NATURAL RESERVOIR ACCOMPONIED BY ITS HYDRATE FORMATION Vestnik Tomskogo gosudarstvennogo universiteta. Matematika $i$ mekhanika [Tomsk State University Journal of Mathematics and Mechanics]. 55. pp. 113-123

DOI $10.17223 / 19988621 / 55 / 10$

Keywords: mathematical model, self-similar solution, porous medium, filtration, gas hydrates, hydrogen sulfide.

Based on the equations of mechanics of multiphase media, a mathematical model of liquid hydrogen sulfide injection into a porous formation saturated with oil and water is developed. To describe the processes of heat and mass transfer in the porous medium, the combined equations including mass and energy conservation laws, Darci's law, and the equation of state are used. 
Self-similar solutions to the problem, in which three characteristic areas are formed in the reservoir, are obtained. In the first area (the nearest region), the porous are saturated with liquid hydrogen sulfide and its hydrate; in the second area (intermediate region), there is water and liquid hydrogen sulfide; and in the third area (distant region), the porous are saturated with oil and water.

Self-similar coordinates of the interphase borders are studied as functions of permeability of the reservoir and its initial pressure. It is established that increase in the initial pressure of the reservoir and decrease in its permeability cause a decrease in the velocity of oil displacement by hydrogen sulfide. It is shown that in conditions of low permeability of the reservoir and high initial pressure, the merge of interphase boundaries could happen. The dependences of critical values of injection pressure, corresponding to a merge of interphase boundaries, on the permeability of the reservoir and its initial pressure are found.

KHASANOV Marat Kamilovich (Candidate of Physics and Mathematics, Sterlitamak Branch of Bashkir State University, Sterlitamak, Russian Federation). E-mail: hasanovmk@mail.ru

\section{REFERENCES}

1. Machel H.G. (2005) Geological and hydrogeological evaluation of the Nisku Q-Pool in Alberta, Canada, for $\mathrm{H}_{2} \mathrm{~S}$ and/or $\mathrm{CO}_{2}$ storage. Oil Gas Sci. Technol. 60. pp. 51-65. DOI: 10.2516/ogst:2005005.

2. Xu T., Apps J.A., Pruess K., Yamamoto H. (2007) Numerical modeling of injection and mineral trapping of $\mathrm{CO}_{2}$ with $\mathrm{H}_{2} \mathrm{~S}$ and $\mathrm{SO}_{2}$ in a sandstone formation. Chemical Geology. 24(3-4). pp. 319-346. DOI: 10.1016/j.chemgeo.2007.03.022.

3. Duchkov A.D., Sokolova L.S., Ayunov D.E., Permyakov M.E. (2009) Otsenka vozmozhnosti zakhoroneniya uglekislogo gaza $\mathrm{v}$ kriolitozone Zapadnoy Sibiri [Assesment of potential of West Siberian permafrost for the carbon dioxide storage]. Kriosfera Zemli-Earth's Cryosphere. 13(4). pp. 62-68.

4. Chuvilin E.M., Guryeva O.M. (2009) Eksperimental'noe izuchenie obrazovaniya gidratov $\mathrm{CO}_{2} \mathrm{v}$ porovom prostranstve promerzayushchikh i merzlykh porod [Experimental investigation of $\mathrm{CO}_{2}$ gas hydrate formation in porous media of frozen and freezing sediments. Kriosfera Zemli-Earth's Cryosphere. 13(3). pp. 70-79.

5. Byk S.Sh., Makogon Yu.F., Fomina V.I. (1980) Gazovye gidraty [Gas hydrates]. Moscow: Khimiya.

6. Shagapov V.Sh., Rafikova G.R., Khasanov M.K. (2016) On the theory of formation of gas hydrate in partially water-saturated porous medium when injecting methane. High Temperature. 54(6). pp. 858-866. DOI: 10.1134/S0018151X16060171.

7. Gimaltdinov I.K., Khasanov M.K. (2016) Mathematical model of the formation of a gas hydrate on the injection of gas into a stratum partially saturated with ice. Journal of Applied Mathematics and Mechanics. 80(1). pp. 57-64. DOI: 10.1016/j.jappmathmech.2016.05.009.

8. Khasanov M.K. (2017) Inzhektsiya zhidkogo serovodoroda v plast, nasyshchennyy neft'yu i vodoy [Injection of liquid hydrogen sulfide in a layer saturated with oil and water]. Vestnik Tyumenskogo gosudarstvennogo universiteta. Fiziko-matematicheskoe modelirovanie. Neft', gaz, energetika - Tyumen State University Herald. Physical and Mathematical Modeling. Oil, Gas, Energy. 3(2). pp. 72-84. DOI: 10.21684/2411-7978-2017-3-2-72-84.

9. Khasanov M.K. (2017) Matematicheskaya model' inzhektsii serovodoroda v poristuyu sredu, nasyshchennuyu neft'yu i vodoy [Mathematical model of hydrogen sulfide injection into a porous medium saturated with oil and water]. Izvestiya Tul'skogo gosudarstvennogo universiteta - Izvestiya Tula State University. 9. pp. 337-345.

10. Shagapov V.Sh., Khasanov M.K., Musakaev N.G., Ngoc Hai Duong (2017) Theoretical research of the gas hydrate deposits development using the injection of carbon dioxide. International Journal of Heat and Mass Transfer. 107. pp. 347-357. DOI: 10.1016/ j.ijheatmasstransfer.2016.11.034. 